Ist jetzt schon bekannt, wann TarMed definitiv eingeführt wird?

M. R. in B.

Die Einführung der definitiven Tarifstruktur Alpha 1.0 soll spätestens auf den 31. Dezember 2001 erfolgen.

Bislang konnten dieselben Leistungen in Kantonen ohne SD zu höheren TP-Werten verrechnet werden als in Kantonen mit SD, so dass interkantonal innerhalb derselben Fachrichtungen die Einkommen angepasst wurden. Nach Einführung des TarMed sollen die Taxpunktwerte nach wie vor kantonal geregelt sein, dies ebenfalls unter Berücksichtigung der SD? Welche Änderungen ergeben sich für $S D$-Ärzte, falls die margenunabhängige Medikamentenabgabe (MUMA) eingeführt wird? Welche Auswirkungen hätte dies auf das
Einkommen von Ärzten, deren Umsatz bis heute zu einem grossen Teil auf der Medikamentenabgabe beruht (z.B. abgelegene Orte ohne Apotheke)? Wird durch die Einführung der MUMA/TarMed die Anwendung einer speziellen Software zur (Medikamenten-)Abrechnung zwingend, ist diese bereits erhältlich und wenn ja, wo?

K. B. in B.

Solange die Medikamentenabgabe margenabhängig erfolgt, wird wohl die kostenneutrale Umrechnung des TPW auf der AL neutral sein. Bei Wegfall der margenabhängigen Medikamentenabgabe wird beim TPW auf der AL wieder entsprechend dazugerechnet werden müssen. TarMed braucht keine spezielle neue Software; die etablierten Softwareanbieter müssen gewisse Vorgaben erfüllen, damit mit TarMed abgerechnet werden kann.

\section{Questions les plus fréquentes sur le TarMed}

Je ne vois pas comment la facturation par voie électronique pourra fonctionner dans le système du tiers garant. Il faudra que j'envoie mes données formatées et codées d̀ un centre fiduciaire. Lequel? Comment pourrai-je le contacter? Qui imprimera les factures et qui les adressera aux patients? Tout cela fonctionnera-t-il déjà dans 5 mois?

$$
\text { J.-L. M. à B. }
$$

Les conditions générales ayant trait au centre fiduciaire dans le cadre du TarMed sont encore en discussion. Dans le système du tiers garant, la facture sera établie par voie électronique et adressée à l'assureur lorsque le patient, qui aura reçu la facture parallèlement sur papier ou par courrier électronique, aura donné son accord soit par téléphone, soit par lettre ou par e-mail. Si vous n'avez pas l'intention d'établir vos factures par voie électronique, vous pourrez les faire parvenir au centre fiduciaire par l'intermédiaire de la Caisse des médecins, de Médipa ou de Médidata, par exemple. Cela dit, le TarMed n'entrera vraisemblablement en vigueur qu'au $1^{\text {er }}$ juillet 2001.
Mon cabinet est situé à environ $20 \mathrm{~km}$ de l'hôpital où j'exerce en tant que médecin agréé. En tant que gynécologue, il n'est pas rare que je n'aie qu'une seule patiente hospitalisée (par visite). Pourrai-je continuer à facturer une indemnité de déplacement (00.0080?) pour les visites à mes patientes ambulatoires ou hospitalisées pour un jour ou l'indemnité de déplacement devra-t-elle faire partie du temps consacré à ces patientes?

D. B. $\dot{a} L$.

Comme il s'agit en l'occurrence d'un second lieu de travail, vous ne pourrez pas facturer d'indemnité de déplacement, à l'instar du tarif cantonal en vigueur.

Une patiente opérée d'un carcinome mammaire est suivie actuellement par une oncologue pour une chimiothérapie. Les contrôles de la formule sanguine (automatisés; Micros/Cobas, p.ex.) sont délégués au médecin de famille ou au gynécologue. Dès lors, comment facturer une prise de sang effectuée par l'assistante médicale, sans consultation médicale? A mon sens, les "Interprétations générales 3" ne sont pas formelles à cet égard. Veuillez vous prononcer.

D. B. $\dot{a} L$.

Dans le TarMed, où se trouvent les prestations de laboratoire, par exemple $\mathrm{Hb}, \mathrm{Ht}$, cholestérol?

$$
\text { P.-A. B. } \dot{a} L \text {. }
$$

La structure tarifaire TarMed ne comprend aucune prestation de laboratoire. Veuillez consulter la Liste des analyses pour la facturation. 
Un médecin suisse en possession d'un diplôme fédéral de médecin et d'une formation complémentaire en médecine manuelle suivie en France peut-il exercer la médecine manuelle en Suisse et avoir ses prestations remboursées par l'assurance de base?

M. K. $\dot{a} G$.

Pour la réponse à cette question, nous vous renvoyons à la Société médicale suisse de médecine manuelle (SAMM), samm@gmx.net.

Je suis spécialiste en médecine générale FMH. Pour l'heure, je suis employée dans un cabinet de médecine générale à la campagne. Au quotidien, je rencontre aussi des cas relevant de la pédiatrie et de la gynécologie, mais les factures ne sont pas établies en mon nom. Avec le TarMed, pourrai-je continuer à facturer ces prestations de cette manière ou devrai-je posséder mon propre numéro de concordat?

D. H. $\dot{a} L$.

A l'avenir, ne pourront être exécutées, respectivement facturées que les prestations pour lesquelles le médecin est détenteur du titre de spécialiste ou de l'attestation de formation complémentaire en question. Le principe des droits acquis garantit que tous les médecins ayant effectué jusqu'ici des prestations de façon régulière et incontestée, prestations auxquelles ils n'auraient plus droit selon la nouvelle valeur intrinsèque qualitative, pourront continuer de le faire.

Comment le matériel de consommation (selon LiMA 34.1.1 compresses de gaze/compresses vulnéraires) pourra-t-il être facturé? Sera-t-il indemnisé selon le chiffre 21 des Interprétations générales du TarMed (<Fr. 200.-) ou pourra-t-il être facturé en supplément selon l'Interprétation générale LiMA? Dans la première hypothèse (Fr. 200.-), il y aurait la possibilité pour le médecin d'établir une ordonnance. La facture de la pharmacie devrait ainsi être réglée de manière séparée (en supplément?) ce qui ne manquerait pas d'engendrer des coûts supplémentaires. Estce bien conforme au tarif?

Helsana à Z.

La réglementation concernant le remboursement du matériel de consommation a été adoptée dans le tarif sous la pression des assureurs (CTM/CAMS) et n'a pas soulevé l'enthousiasme du corps médical. Il va de soi que la possibilité d'établir une ordonnance et de se rendre à la pharmacie existe. Le matériel de consommation serait ainsi remboursé. La FMH est déjà intervenue concernant cette interprétation et ne peut que se réjouir qu'une caisse-maladie importante relève une telle ineptie. Nous vous suggérons donc de vous adresser aux responsables du CAMS en matière tarifaire.
Concernant le chapitre sur la psychiatrie, et plus particulièrement dans le 02.02 prestations non médicales en psychiatrie, il est dit que les prestations sont indemnisées 6 heures par semaine. Faut-il comprendre que lorsque plusieurs thérapeutes, psychologues, infirmières, travailleurs sociaux, etc., sont actifs, le temps total pour l'ensemble du personnel est limité à 6 heures par semaine ou que cette limite est pour chaque thérapeute?

\section{R. C. $\dot{a} P$.}

Il existe diverses prestations soumises à une limitation. Mais, pour certaines d'entre elles, cette limitation est accompagnée de la remarque suivante (cf. interprétation technique): "Cette restriction ne s'applique pas aux spécialistes établissant leurs factures par voie électronique».

Pour les prestations ne comportant pas la remarque ci-dessus, les limitations doivent être considérées comme fixes et être respectées comme telles. En admettant qu'après un an d'application du TarMed, les coûts soient plus élevés que prévus, et cela malgré la neutralité des coûts escomptée, devra-t-on s'attendre (pour l'ensemble du corps médical) à des demandes de restitution de la part des caisses-maladie?

\section{J. B. $\dot{a} L$.}

Il n'y a vraisemblablement pas à craindre de telles demandes de la part des caisses-maladie. Si les coûts s'avéraient plus élevés que prévus avec le TarMed, il y aurait lieu de renégocier la valeur du point. Si, au contraire, les coûts devaient baisser, il est clair que les caisses-maladie n'accorderaient pas non plus de compensation aux médecins.

En tant qu'interniste porteur de la sous-spécialité "cardiologie", pourrai-je facturer mes prestations aussi bien en tant que médecin de premier recours (valeur intrinsèque 5) que comme cardiologue (valeur intrinsèque 8) pour un seul et même patient? Dans la négative, comment devrai-je saisir mes nombreuses prestations de base (service d'urgence, par ex.)?

$$
\text { J. F. } \dot{a} L \text {. }
$$

Une prestation ne pourra être facturée que par un spécialiste satisfaisant aux exigences de valeur intrinsèque de cette prestation (titre de spécialiste, attestation de formation complémentaire, p. ex.). Cela dit, un cardiologue (valeur intrinsèque 8) facturera une consultation de base, par exemple, au même prix qu'un médecin de premier recours (valeur intrinsèque 5). (Autrement dit, les prestations seront remboursées de la même manière à tous les médecins ayant le droit de les facturer). 
Depuis 1994, j'ai un cabinet où j'exerce exclusivement la psychiatrie et la psychothérapie, sans être porteur du titre FMH. Pourrai-je obtenir un titre d'équivalence pour la psychiatrie et la psychothérapie et continuer à facturer les positions correspondantes dans le TarMed? Quand devra-t-on demander ce titre et l'attestation de formation complémentaire?

R. N. $\dot{a} B$.

Le principe des droits acquis garantit que tous les médecins ayant effectué jusqu'ici, de façon régulière et incontestée, des prestations auxquelles ils n'auraient plus droit selon la nouvelle valeur intrinsèque qualitative, pourront continuer à les fournir.

Avec le TarMed, les médecins des cantons connaissant la propharmacie auront-ils toujours un intérêt financier à pratiquer la vente de médicaments lou ne seront-ils indemnisés que pour leurs frais)?

M. W. $\dot{a} Z$.

Nous ne sommes pas en mesure de vous répondre, l'OFAS étant muet à ce sujet.

Avec une formation postgraduée de 2 ans selon la LAMal, pourrai-je facturer mes prestations d'ergométrie si je puis prouver en avoir exécuté 300 de manière autonome durant ma formation postgraduée? Quelles prestations un interniste FMH pourra-t-il facturer en plus par rapport à un titulaire d'une formation postgraduée de 2 ans selon la LAMal?

M. K. $\dot{a} S$.

L'interniste FMH aura le droit de facturer l'ensemble des prestations avec valeur intrinsèque qualitative "toutes" ainsi que les prestations du médecin de premier recours. Il lui sera également possible de continuer à facturer d'autres prestations selon le principe des droits acquis. Le concept de la valeur intrinsèque est déterminant à cet égard.

Qui est compétent pour attester la valeur intrinsèque qui permettra de facturer la prestation correspondante?

M. K. $\dot{a} S$.

La valeur intrinsèque dépend du titre de spécialiste ou, le cas échéant, de l'attestation de formation complémentaire/du certificat d'aptitude technique. Les droits acquis entrent eux aussi en ligne de compte. Tout cela figure dans le concept de valeur intrinsèque de la FMH/TarMed qui n'a malheureusement pas encore pu être publié. La version définitive de ce papier ne sera disponible qu'après la publication de l'Ordonnance sur la libre circulation des personnes. S'agissant des droits acquis, le médecin pourra continuer à faire état des prestations qu'il entend continuer de facturer. Il sera inscrit par la FMH.
Sait-on déjà quand TarMed sera mis en vigueur?

M. R. $\dot{a} B$.

La mise en vigueur de la structure tarifaire définitive, alpha 1.0, devrait avoir lieu au 31 décembre 2001 au plus tard.

Jusqu'ici, pour les mêmes prestations, la valeur du point était plus élevée dans les cantons sans propharmacie que dans les autres, si bien que sur le plan intercantonal les revenus des mêmes disciplines ont dû être adaptés. Après la mise en vigueur du TarMed, la valeur du point sera-t-elle toujours fixée au niveau cantonal, et cela en tenant compte également de la propharmacie? Que va-t-il se passer pour les médecins propharmaciens en cas d'introduction de la distribution de médicaments indépendante de la marge? Quelles en seront les conséquences sur les revenus des médecins dont le chiffre d'affaires repose pour une grande part sur la distribution de médicaments (lieux éloignés sans pharmacie, p.ex.)? L'introduction $d u$ TarMed et de la distribution de médicaments indépendante de la marge impliquera-t-elle un logiciel spécial pour la facturation des médicaments? Le cas échéant, est-il déjà disponible et si oui auprès de qui?

K. B. $\dot{a} B$.

Aussi longtemps que la distribution des médicaments dépendra de la marge, le calcul de la valeur du point tarifaire n'aura pas d'effet sur la prestation médicale (PM). En cas de suppression de ce mode de distribution, il faudra tenir compte de l'effet correspondant sur la PM pour le calcul de la valeur du point. Le TarMed n'aura pas besoin d'un nouveau logiciel. Des conditions bien précises seront posées aux fournisseurs de logiciels pour permettre la facturation au moyen du TarMed. 